\title{
TOWARDS SECURITY ARCHITECTURE FOR FUTURE ACTIVE IP NETWORKS
}

\author{
Dusan Gabrijelčič \\ and Arso Savanović \\ and Borka Jerman Blažič \\ Institute Jožef Stefan \\ Ljubljana, Slovenija \\ dusan@e5.ijs.si, arso@e5.ijs.si, borka@e5.ijs.si
}

\begin{abstract}
Active networks allow user-controlled network programmability. A security framework has to assure that an active networks infrastructure will behave as expected and will efficiently deal with malicious attacks, unathorized attempts to execute active code etc. We present here a security architecture that is designed within the FAIN project and aims at supporting multiple heterogeneous execution environments. We argue for the pros and cons as well as why we have selected the specific components and also take a look at their interworking in order to provide the security services to the execution environments our active network node hosts.
\end{abstract}

Keywords: Active Networks, Security Architecture, Active Packets, Security Management

\section{INTRODUCTION}

Active networks enable their users to program network elements by injecting the code in the network which provides new services for the users in the network. Such flexibility raises many security concerns. Security architecture as described in the following paper, dealing with these concerns, is designed and developed in a FAIN [2] project.

FAIN project goal is to provide open, flexible, high-performance and secure active network infrastructure. Such infrastructure consist of interconnected active and passive nodes. Active nodes provide support for various active networking technologies and are able to support various active networking applications in control, management and data plane. Core of FAIN active network (AN) is active network node (ANN).

The original version of this chapter was revised: The copyright line was incorrect. This has been corrected. The Erratum to this chapter is available at DOI: 10.1007/978-0-387-35612-9_23 


\section{FAIN ACTIVE NODES}

The FAIN Reference Architecture consists of Active Applications (AA), Virtual Environments (VE), Execution Environments (EE) and Node Operating System (NodeOS).

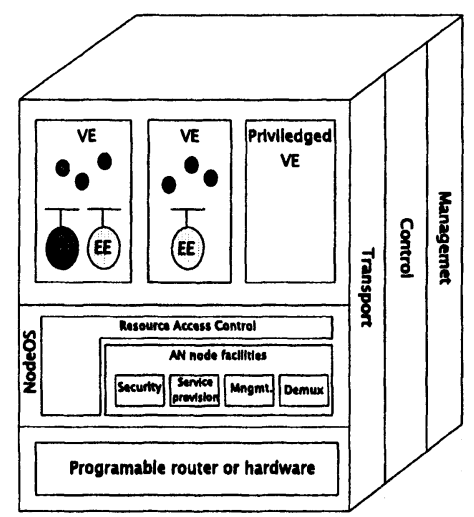

Figure 1. FAIN Active Node reference architecture

Active Applications/Services are active code executed or running in Active Nodes. Active code enable, support or enhance communication between active network users.

Execution Environments are environments in the active node where the active code is executed. Execution environments represent programming model that active code comply to and abstractions on which Active Applications can be build. A privileged EE in privileged Virtual environment (PVE) manages and controls the Active Node and it provides the environment where network policies are executed. Multiple and different types of EE are envisaged in FAIN. EEs are classified into Virtual Environments (VEs), where services can be built and interact with each other. VEs represent principals on the node. Interconnected VEs form a truly virtual network.

NodeOS is an active node operating system and provides basic abstractions for network communication, resources and inter EE communication. It manages node resources which are accessible through NodeOS APIs. NodeOS functionality is divided into several subareas, which are described in the following paragraphs with short emphasis also on their role in overall node security:

Demultiplexing/Multiplexing subsystem. It filters, classifies and diverts active or passive packets. Flows of packets arrive at the node and they should be delivered to the VE and consequently to the service in- 
side the VE they are destined for. Demultiplexing represents the entry point of the node for packets, which can be seen as entities that request processing on the node and will use node resources. At this point node is protecting its own resources. In multiplexing subsystem packets are sent towards next hops; here, well behaved active node should protect other nodes.

Resource Control Framework (RCF). Through resource control resource partitioning and access to the resources is provided. VEs are guaranteed that consumption stays within the agreed contract during an admission control phase static or dynamic. It provides also guaranteed share of resources to the node subsystems and privileged virtual environment.

Active Service Provisioning (ASP). As flexibility is one of the requirements for programmable networks partly realised as service deployment either on the fly or static, the NodeOS must support it. ASP must work closely with Security subsystem in the way that only code and services that meets certain security criteria can be made available on the node.

Management subsystem. Management subsystem provides interfaces that are meaningful on the NodeOS level. Among them there are interfaces that enable the management system to insert security policies and register entities as principals on the active node.

Security subsystem. Security subsystem is a collection of needed security services, mechanisms and elements that are core of the security architecture. Together with other subareas of the node this subsystem has to fulfill the security goals as stated in the next section.

\section{FAIN SECURITY ARCHITECTURE: GOALS AND SCOPE}

FAIN general active networking model follows the general active networking model as described in [3]: the primary unit of communication is a packet, primary goal is communication and not computation in the network, ANN are interconnected with various packet-forwarding technologies, AN consist of multiple domains controlled by different administrations and end systems and intermediate systems have same architectural components. To this common starting point we can add the following general AN properties: not all nodes in the network need to be active, packets used for communication can be both active and "passive", AN can provide per user service in the network by injection of the code in the network both in-band or out-of-band, active application can keep state in the network or in the packets, active packets can legally change in the network, there is no unified address space in AN 
and packet resource consumption in the $\mathrm{ANN}$ and $\mathrm{AN}$ is hard to predict and control.

All AN properties as stated, have serious security consequences on AN and ANN operation. They raise many security related questions: where the packet has come from, is it the same as when sent from data origin, who has send the packet, can the code that packet address/carry be safely run on the node, does it access privileged node resources and interfaces, how many resources can/will the packet consume on the node etc. To tackle these questions we have identified a set of possible security architecture goals. From this set we have focused in initial phase only on few, high priority ones, that protect the AN infrastructure from intentional or unintentional misbehaving of AN users. Our primary security architecture goals are authentication, authorization, policy enforcement, active code and packet integrity, code verification and audit.

Because FIAN active node supports multiple and different execution environments and technologies we have designed and focused on security architecture on NodeOS level as common denominator of the node.

\subsection{Authentication, authorization and policy enforcement}

FAIN ANN is essentially a multi-user computing system. As in any such system, enforcement of access control is a requirement of high significance within every FAIN ANN. On the other hand, FAIN aims at developing a flexible system. In order to achieve the desired level of granularity and flexibility we decompose access control into authentication, authorization and policy enforcement.

Authentication is a process of verifying an identity claimed by or for a system entity. Authentication is a basic security service that other services depend on. From a range of solutions based on either symmetric or asymmetric cryptography we have chosen to provide authentication service on the basis of digital signature mechanism. The main reason for this decision is that the active packet can pass many nodes in the network where its data origin is authenticated. Proper authentication solutions that require handshake between active packet source and every possible active node in the packet path are too costly, even though they can be amortized over time. Using digital signature mechnisms for authentication requires, that every user as well as every active node has at least one valid key pair, and existence of a public key infrastructure.

Authorization is a process of authorizing user access to certain node resources. Resources can be hardware like CPU, memory, storage and link bandwidth or functional like special purpose files, routing tables, 
policy and credentials entries and databases etc. All node resources are accessible through set of certain node interfaces. Authorization decision is made based on information about subject requesting a resource, object being accessed, action requested and possible environment of the request. To support such decision relevant security policies must be available in the node policy database and the security contexts of the subject and the object have to be built and kept unforgeable on the node. Authorization decision is then enforced in node enforcement engines.

Policy enforcement is a task of many node enforcement engines which resides in the node subsystems. The task is twofold: upon request to access certain node interface the engine suspends the request, collects the relevant information (object, subject, action and environment) and asks for authorization decision. When authorization decision is made, the enforcement engine either discards or resumes the request. In both cases the resulted decision and enforcement engine action can be audited.

\subsection{Active code/packet integrity}

Active code and packet integrity is an important issue and thus high priority goal in FAIN AN. In both in-band (code and data in packet) and out-of-band (code is provided separately) active network approaches the content of the active packet has high impact on the active application. Every change of the packet like replacing or modifying its content, possible cut and paste or replay attacks can have unpredictable consequence of the behavior of the active application as a whole. Providing integrity service for active packet traversing many nodes is also an issue. Active packets can carry a state or can change (packet or in-band code) in the network. This means that the data integrity calculated at the originating node can be verified only if the data that the integrity service covers has not changed in between the source and the point of its verification. Logical consequence of the stated is, that the integrity service has to be provided separately for the variable and static data in the packet. Packet integrity is closely related to the authentication as presented in section 3.1. There can be no direct authentication if the integrity property of the data which origin is being authenticated is not provided as well. Digital signature used for authentication provides also integrity property for the data but only for the static parts of the packet which the digital signature covers. To protect the packet's variable content when the packet traverses the network we use a keyed hash as protected integrity token and sequences for replay attack prevention in between pair of neighbor active nodes. Such integrity service requires shared secret between a pair of neighbor nodes; this secret can be es- 
tablished either via management system or dynamically with suitable key exchange protocol. It has to be noted that such integrity protection protects data only between two neighbor active nodes; trust in integrity of data that has passed many nodes means that we trust all the nodes, the packet has already passed, which can be a weak assumption from security point of view.

The last observation can be specially true for the active code if inband approach is used. In this case data and code can be mixed together and the "code" can change on every hop. Integrity of such code can be protected only per hop and not between source and destination.

\subsection{Code verification}

Protecting the active code integrity is a first step to ensure non- modification of the transient code. However this is considered pretty basic and we need to go beyond that in order to achieve higher level of security. The active code has to be somehow marked and tightly coupled with one or more entities, based on which further security decisions can be made. As an alternative, code in conjunction with $\mathrm{EE}$ has to exhibit certain properties which can be verified on the node.

Verification can enable us to trust to some extent that the active code will behave safely and properly and that we can have some guarantees on its resource usage on the node and in the network. But we shall say in general that verification provides only enhanced trust in proper and safe code execution, which is usually not related to the trust in the entity on behalf of which the code is executing. Besides the root of trust is in many cases different for the code and the packet that triggers the code on the nodes. Code verification can help an ANN decide whether to run the newly received code. If the code fails the verification test, it is not trusted and it is dropped or alternatively it can run in an $\mathrm{EE}$ with minimal facilities available. In the latter case the $\mathrm{EE}$ is the same one that will be used to run anonymous active code. Broadly we can divide verification in two groups: first, in which we trust in an entity in the system that enable us to believe in some code properties and second, belief in code properties by design (safe languages or/and interpreters) or additional means like proof carrying code [7]. In the first, general case, node can trust user, network owner, manufacturer, code packager, code repository or dedicated organization, which can in various ways guarantee some code properties. Code can be source inspected, tested or proved otherwise to work as claimed. To enable trust in an entity we use digital signature mechanisms and code certificate, in which the claimed properties or conditions of use of the code can be stated and 
verified prior the code installation/usage directly on the NodeOS level. In the second case we have to trust EEs to properly verify the code prior and/or during its execution.

\subsection{Audit}

The Audit Manager component is an integrated part of the security architecture. Via this component all events occurring from the usage of the security subsystem are implicitly logged for further future usage. It also provides an interface to explicitly log any other events coming from other parts of the FAIN architecture in a clear and homogeneous way. Modern computer systems do not emphasize enough on the significance of the audit facilities. However audit tools help in realizing possible security leaks (or even preventing some) and make sure that mistakes are not repeated.

\section{FAIN SECURITY ARCHITECTURE}

Figure 2 depicts a FAIN active network node with basic security architecture components. As depicted, FAIN security architecture roughly comprises three parts: security subsystem, other ANN security components, and external security support facilities. Note that the scope of initial FAIN security architecture does not include EE layer of FAIN ANN architecture.

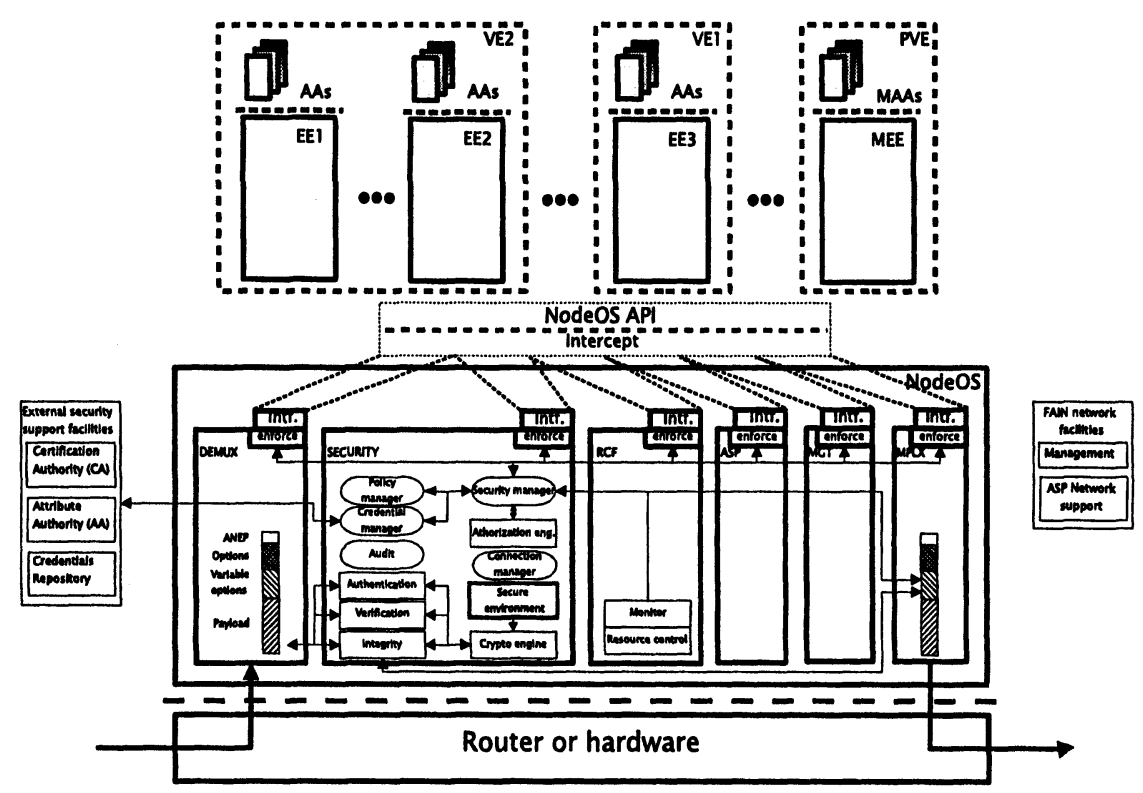

Figure 2. FAIN security architecture 


\subsection{Security subsystem}

Most of security critical decisions are made by security subsystem, which is one of several subsystems within an ANN. The Security subsystem is also responsible for management of security critical data, such as encryption keys, credentials, and policies.

This subsystem is the core of FAIN security architecture and includes the following components:

Crypto Engine: performs the actual cryptographic operations, such as symmetric encryption/decryption, asymmetric encryption/decryption, and hashing. It implements various cryptographic algorithms, which are used by other components in the security subsystem.

Secure Environment (SE): in a secure fashion stores various encryption keys, which are required by crypto engine. For example, SE stores ANN's public key pair (private and public key) and all symmetric keys that an ANN shares with its AN neighbors (one per neighbor).

Connection Manager: is used to manage secure associations with neighbor AN nodes. Associations can be configured manually or their configuration can be supported by automatic management and by triggering a key exchange protocol with neighboring ANN.

Integrity Engine: checks the integrity of active packets and active code. It depends on integrity protection data contained within an active packet and on crypto engine to do the necessary cryptographic operations.

Verification Engine: performs code verification (at NodeOS level), if any. It may depend on special data contained within an active packet and on crypto engine to do the necessary cryptographic operations.

Authentication Engine: verifies the authenticity of active packets. It depends on authentication data contained within an active packet and on crypto engine to do the necessary cryptographic operations.

Enforcement engines: which intercept requests to NodeOS API interfaces and pass them with request specific information to security manager. Security manager responds with authorization decision and enforcement engines enforce this decision. They are distributed and not present only in security subsystem.

Security Manager: accepts request from enforcement engines, collect request related credential information, accessed object policy information and ask authorization engine for authorization decision. This decision is passed back to enforcement engines. Provides NodeOS interfaces for managing AN users related credentials and security policies. It can be used also for caching of the authorization decisions. 
Authorization Engine: is responsible for making a decision whether a given user request to execute specific action or to access/manipulate particular object within an ANN is authorized or not. Authorization engine provides this "service" to all enforcement engines in an ANN.

Policy Manager: when asked by the security manager, searches policy $\mathrm{DB}$ and returns all security policies, that are relevant for a particular request, which is currently subject to authorization. It also provides facilities for editing entries in policy $\mathrm{DB}$, either manually by an authorized user, or automatically, i.e. download policies from a centralized policy server.

Credential Manager: when asked by authorization engine, searches credential DB and returns all credentials, that are relevant for a particular request, which is currently subject to authorization. It also provides facilities for editing credential database, either manually by an authorized user, or automatically, i.e. search and download credentials from an external credential repository.

Audit: will be the place where all security architecture's components audit their function in order to be used later in resolution of problems or even to make decisions. E.g. an Intrusion Detection System would use a view of the audit DB in order to recognize attacks against the system. The audit could be also distributed for survivability reasons.

\section{TOWARDS IMPLEMENTATION}

The basic scenario we had in mind developing security architecture was an active packet passing one or more active nodes and triggering execution on these nodes. While executing or processing the packet, the active code on the nodes can access privileged NodeOS interfaces.

To enable security architecture operation as described in section 3 and to support basic scenario, we have chosen the ANEP packet header [6] as a carrier of needed information for integrity and access control. Basic ANEP options that support integrity, authentication and basic resource usage control are shown in the figure 3. Hop by hop option (3b) consist of KeyId, 64 bit value uniquely identifying security association, 64 bit sequence field and HMAC field holding keyed hash as defined in RFC2104 [11]. Keyed hash covers entire ANEP packet except keyed hash value itself. At the moment security associations are setup via management system; we are developing set of protocols that will support dynamic secret key exchange, sequence synchronization and discovering of the first hop active node.

The credential option (3c) was designed as simple four-tuple option; there can be multiple such options in the packet for additional flexibility. 


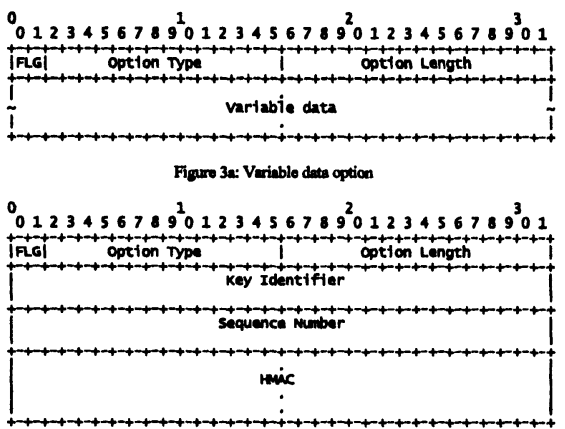

Figure 3b: Hop by Hop option

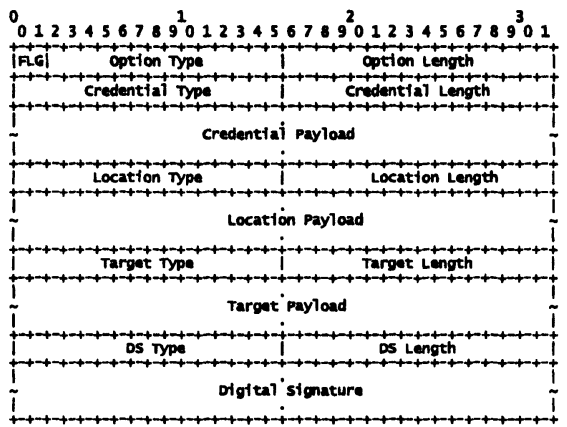

Figure 3e: Crodertial option

Figure 3. ANEP packet options

Credential as designed can be X.509 certificate, X.509 attribute certificate [1] or Keynote credential [8]. Each credential option is validated and signatures verified prior the packet triggers execution on the node and the target field in this option help node to use credentials only in administrative domains, nodes or node subsystems as intended by packet sender or administrative domain boundaries controllers.

Variable option (3a) is meant as a place where active applications can store variable data and is a consequence of integrity discussion in the section 3.2. While is hard to adapt existing approaches to support such option, newly developed will support it out of the box.

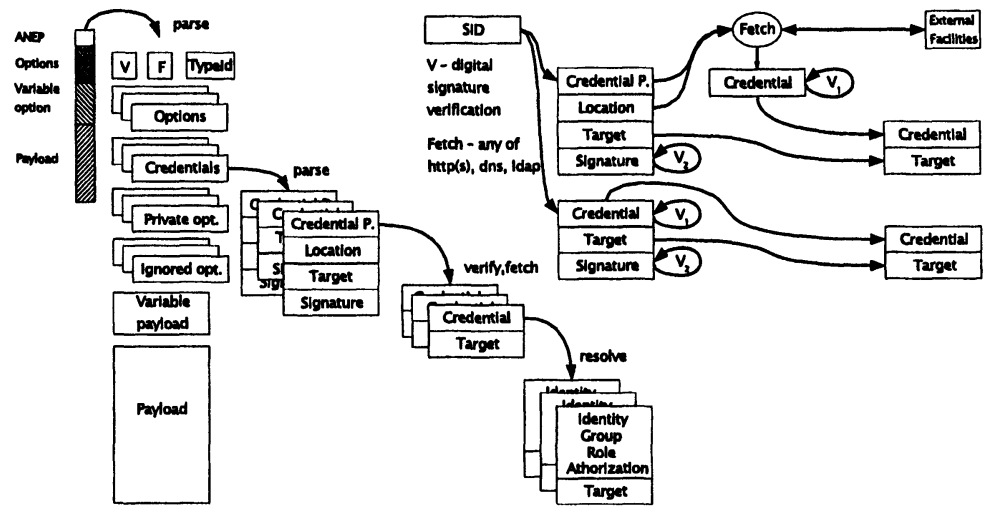

Figure 4. Active packet, identity/role resolving, credentials validation and signature verification 
The figure 4 shows the basic active packet already processed on the active node (left) and process of verification of the one or more attached credentials (right). Credentials can be carried in the option or the option carries the location of the credential; in the second case, the credential has to be obtained from remote repository. In both cases we validate the credential and the possible certification path. With the public key obtained from validated credential, digital signature of the static part of the packet is verified. The next step is resolving identity, group, role or authorization information from the credential. This information is then used in the authorization decisions while the packet (code) access the domain or privileged node interfaces. Domain on the node is a set of related processes/threads that perform certain service for or on behalf of the entity.

The crucial issue on the node regarding access control is security context of the request (packet) passing the node. While the context on the node is defined by entity properties (identity, role or group) and domain information, such context has to be transfered between nodes. While the figure 4 does show how entity information can be transfered through the network, it doesn't show domain related part. Domain is hidden in VE identifier and active code related identifiers. These identifiers must match the domain the packet is destined for.

Policies used in the process of the providing authorization decision are set up on the node by the FAIN management system.

\section{RELATED WORK}

FAIN aims to develop a heterogeneous ANN, allowing coexistence of various technologies that enable installation and execution of active code within an ANN. Consequently, FAIN security architecture is aimed at providing a more general solution which provides necessary protections for such an heterogeneous system. This is reflected by the fact that security architecture we have presented does not incorporate details of specific EEs that exist in the FAIN ANN. Its goal is to be as EE independent as possible and provide a common set of basic security services required by all AN enabling technologies. Some research projects on active networks have already tried to tackle the issue of security [5, 12]. Contrary to FAIN, all these approaches are tied to specifics of particular model of programmability. When designing a more general AN security architecture, which is the case in FAIN, these specifics can not be assumed. Java Security Architecture [9] proved to be useful for AN security, but again it is technology specific and it also has some drawbacks [10]. There has also been some more general work on AN security [4] 
which has also working specific implementation [13]. But the general work is still in draft stage.

\section{CONCLUSIONS}

We have presented in this paper a security architecture for future IP active networks as it is done in the context of FAIN project. We try to tackle the high priority security requirements such as authentication, authorization, policy enforcement, active code and active packet integrity and verification and last but not least audit. We have analysed the main design decisions that we have taken and the reasons why we decided to follow them. Subsequently we have presented the components of a security architecture that will be used by multiple heterogeneous execution environments within the same active node. We also provide a look in the interworkings of the architecture and its decision-making logic. A prototype implementation of the presented active network security architecture is currently under development, which will be used for exploring the advantages and drawbacks of our approach.

\section{ACKNOWLEDGEMENTS}

This paper describes work undertaken and in progress in the context of the FAIN - IST 10561, a 3 year project during 2000-2003. The IST program is partially funded by the Commission of the European Union. The FAIN consortium consists of University College London - UK, Jozef Stefan Institute - Slovenia, NTUA - Greece, Universitat Politecnica de Catalunya - Spain, Deutsche Telekom Berkom - Germany, France Telecom/CNET - France, KPN - The Netherlands, Hitachi Europe Ltd. UK, Hitachi, Ltd. - Japan, Siemens AG - Germany, ETH - Switzerland, Fraunhofer FOKUS - Germany, IKV++ GmbH - Germany, INTERGAsys - Spain, University of Pennsylvania - USA.

\section{References}

[1] ITU-T X.509 (2000) - ISO/IEC 9594-8:2000 - information technology - open systems interconnection -the directory: Public-key and attribute certificate frameworks. Final Draft International Standard, June 2000.

[2] Fain project home page, http://face.ee.ucl.ac.uk/fain/.

[3] Active Network Working Group. Architectural Framework for Active Networks Version 1.1, december 2001.

[4] Active Networks Security Working Group. Security Architecture for Active Nets, maj 2001.

[5] D. Scott Alexander, William A. Arbaugh, Angelos D. Keromytis, and Jonathan M. Smith. Security in active networks. In Secure Internet Program- 
ming: Issues in Distributed and Mobile Object Systems, Lecture Notes in Computer Science State-of-the-Art. Springer-Verlag, 2000.

[6] D. Scott Alexander, Bob Braden, Carl A. Gunter, Alden W. Jackson, Angelos D. Keromytis, Gary J. Minden, and David Wetherall. Active network encapsulation protocol (anep). Active Network Group draft, july 1997.

[7] Andrew W. Appel, Edward W. Felten, and Zhong Shao. Scaling proof-carrying codeto production compilers and security policies. whitepaper, January 1999.

[8] Matt Blaze, Joan Feigenbaum, John Ioannidis, and Angelos D. Keromytis. RFC 2704: The KeyNote trust-management system, version 2, september 1999.

[9] Li Gong. Java security architecture (JDK1.2). Technical report, Sun Microsystems, oktober 1998.

[10] Active Networks Working Group. SANTS Security Overview, May 2000.

[11] H. Krawczyk, M. Bellare, and R. Canetti. Hmac: Keyed-hashing for message authentication. RFC2104, Informational, februar 1997.

[12] Zhaoyu Liu, Prasad Naldurg, Seung Yi, Roy H. Campbell, and M. Dennis Mickunas. Seraphim: Dynamic interoperable security architecture for active networks. In Proceedings OpenArch 2000. University of Illinois, Urbana-Champagain, marec 2000.

[13] Murphy S., Lewis E., Puga R., Watson R., and Yee R. Strong security for active networks. In IEEE OPENARCH 2001 Proceedings, april 2001. 